\title{
The Use of Standardized Reporting and Time-in-Range in the Management of Diabetes: A Consensus Report
}

\author{
Saud Al Sifri', Adnan Alshaikh ${ }^{2}$, Bassam Bin-Abbas ${ }^{3}$, Eman Sheshah4, Fahad Al Sabaan ${ }^{5}$, \\ Mohammed Al-Dawish', Mohammed Al-Dubayee7, Naweed Al-Zaman', Raed A. Al-Dahash", \\ Saad Alzahrani10, Emad R. Issak ${ }^{11^{*}}$ \\ ${ }^{1}$ Endocrinology and Diabetes Department, Alhada and Taif Armed Forces Hospitals, Taif, Saudi Arabia \\ ${ }^{2}$ Pediatrics Department, King Abdulaziz Medical City-Ministry of National Guard Health Affairs, King Khalid National Guard \\ Hospital, Jeddah, Saudi Arabia \\ ${ }^{3}$ Pediatrics Department, King Faisal Specialist Hospital and Research Center, Riyadh, Saudi Arabia \\ ${ }^{4}$ Endocrinology Department, King Salman hospital, Riyadh, Saudi Arabia \\ ${ }^{5}$ Endocrinology Department, Security forces hospital program, Riyadh, Saudi Arabia \\ ${ }^{6}$ Endocrinology Department, Prince Sultan Military Medical City (PSMMC), Riyadh, Saudi Arabia \\ ${ }^{7}$ Pediatrics Department, Ministry of National Guard Health Affairs-Riyadh, Riyadh, Saudi Arabia \\ ${ }^{8}$ Endocrinology Department, Taibah University, Madina, Saudi Arabia \\ ${ }^{9}$ Internal Medicine Department, King Abdulaziz Medical City-National Guard, Riyadh, Saudi Arabia \\ ${ }^{10}$ Endocrinology Department, Medical Director, OEMC, King Fahad Medical City, Riyadh, Saudi Arabia \\ ${ }^{11}$ Internal Medicine Department, Faculty of Medicine, Ain-Shams University, Cairo, Egypt \\ Email: *dr.emad.r.h.issak@gmail.com
}

How to cite this paper: Al Sifri, S., Alshaikh, A., Bin-Abbas, B., Sheshah, E., Al Sabaan, F., Al-Dawish, M., Al-Dubayee, M., Al-Zaman, N., Al-Dahash, R.A., Alzahrani, S. and Issak, E.R. (2021) The Use of Standardized Reporting and Time-in-Range in the Management of Diabetes: A Consensus Report. International Journal of Clinical Medicine, 12, 316-327.

https://doi.org/10.4236/ijcm.2021.128028

Received: July 1, 2021

Accepted: August 2, 2021

Published: August 5, 2021

\begin{abstract}
Introduction: The exhaustion of healthcare resources due to the rising prevalence in Saudi Arabia mandates the search for each method that can help in better control of diabetes. Methods: The gathered task force gathered to develop an explicit, evidence-based consensus for the use of time-in-range targets as guidance for better glycemic control while using continuous glucose monitoring (CGM). This article has the recommendations of this expert panel. Results: $\mathrm{HbA1c}$ and self-monitoring blood glucose (SMBG) are not enough to detect blood glucose (BG) fluctuations on a daily basis. The incorporation of technology like FreeStyle Libre with its applications like Libre View is now used in many institutes in Saudi Arabia. This system is comprehensive and has all the standardized metrics needed. However, training and support are always needed. Barriers and challenges include the awareness \& experience of the technology, the time barrier, the patients' barriers, the technical barriers, and of course, the availability barrier. All the barriers and challenges should be dealt with by designing new training programs. Conclusion: The expert
\end{abstract}


Copyright $\odot 2021$ by author(s) and Scientific Research Publishing Inc. This work is licensed under the Creative Commons Attribution International License (CC BY 4.0).

http://creativecommons.org/licenses/by/4.0/ panel recommended using CGMs technology in people with type 1 diabetes (T1DM) children and adults, type 2 diabetes (T2DM) on multiple insulin injections, gestational diabetes (GDM) who need further glycemic control, and those at high risk for hypoglycemia. In addition, we recommend using them for a short period for those who require intensive BG control or during acute illness or stress. In addition, Ambulatory Glucose Profile (AGP) could be used as an educational tool for any individuals with DM to study the impact of certain elements of lifestyle modifications on their immediate BG level.

\section{Keywords}

Diabetes, Time in Range, Glycemic Control, HbA1c

\section{Introduction}

Worldwide, by the year 2045, diabetes mellitus (DM) prevalence is expected to be $9.9 \%$, with a total number of 629 Million [1]. The Kingdom of Saudi Arabia (KSA) has a rising prevalence of DM [2], with the consequent exhaustion of healthcare resources.

In 2019, the American Diabetes Association (ADA) coined and published its first recommendations for the time-in-range (TIR) targets to guide those who help in the management of DM as well as people with DM achieve better glycemic control by the utilization of the continuous glucose monitoring (CGM) [3]. The consensus panel included researchers from all geographic regions to ensure that the recommendations can be generalizable [4].

A panel of experts in DM was gathered to generate a clear, evidence-based consensus for the use of TIR targets as guidance for better glycemic control while using CGM. This manuscript presents the recommendations of this task force.

\section{Available Metrics for Blood Glucose Monitoring}

Fasting blood glucose (FBG) level, postprandial blood glucose (PPBG) level, and random blood glucose (RBG) level-metrics for blood glucose (BG) monitoring -were used once for the diagnosis and management of DM. However, they give only a snapshot of the glycemic status at a certain point in time. Glycated hemoglobin (HbAlc) and fructosamine were also introduced as metrics for glycemic control over a duration ranging from two weeks to three months. The introduction of self-monitoring blood glucose (SMBG) helps in glycemic control daily; however, to reflect the actual status, it should be carried out at least seven times per day, which is sometimes not practical. All those lead to the evolution of continuous blood glucose monitoring systems (CGMs), particularly in those people who are in intensive insulin therapy. Consequently, new metrics have been developed to reveal new insights into the short-term glucose dynamics; this is the topic of this consensus [5] [6]. 


\subsection{Glycated Hemoglobin (HbA1c) Is Not Enough Metric for Glycemic Control on a Daily Basis}

Elevated $\mathrm{HbAlc}$ is a significant contributor to complications in people with type 1 DM (T1DM), as confirmed by the DM Control and Complications Trial (DCCT). In addition, the UK Prospective Diabetes Study (UKPDS) confirmed how the control of BG affects health outcomes in type 2 DM (T2DM) [7] [8].

However, HbA1c has its limitations. First, it does not show the glycemic level and variability daily, as it just shows an average level of BG for the last three months.

Second, $\mathrm{HbAlc}$ is inaccurate in people with anemia, hemoglobinopathies, and pregnancy [9] [10] [11] [12]. In addition, it does not reveal the rapid changes in BG levels daily; thus, adjustment of therapy is not easy. Moreover, there is a racial difference in the accuracy of HbAlc because of different glycation rates [9]. Therefore, even though $\mathrm{HbAlc}$ has been evidenced valuable measure and validated as a risk factor for DM complications, it seems not helpful for glycemic control on a personal level as it reflects only a piece of the severity of hyperglycemia and glycemic variability are contributing to the pathogenesis of complications [13] [14].

\subsection{Self-Monitoring Blood Glucose (SMBG) Also Has Its Limitations as a Metric for Glycemic Control}

SMBG has been associated with better management in T1DM and T2DM. Nevertheless, it requires a finger-stick and it only gives a snapshot for one point in time; therefore, it does not show the trend or the rate of change of BG levels. Thus, using it alone may result in improper treatment decisions. Moreover, it often fails to detect hypoglycemia, either nocturnal or asymptomatic [15]-[22].

\section{The Advent of Continuous Blood Glucose Monitoring Systems (CGMs)}

The search for new methods for BG monitoring was continuous to address the limitations in $\mathrm{HbA1c}$ and SMBG, leading to the development of real-time CGM (rtCGM) and flash glucose monitoring (FGM). The former tracks the glucose level uniformly, providing real-time measurements, while the latter, at the time of checking, shows continuous glucose measurements retrospectively. Both types facilitate monitoring of the time spent in the target glycemic range; TIR.

Nevertheless, only rtCGM can warn users if their BG level is trending toward hypoglycemia or hyperglycemia, while FGM requires scanning of the sensor to reveal these trends, where newer generations of FGM are available with optional alarm functionality. Plentiful studies have demonstrated that the use of CGM improves both glycemic control and quality of life in different populations with T1DM or T2DM. In addition, one meta-analysis has shown that the frequency and persistence of its utilization of rtCGM are directly correlated to its benefit [9] [23]. Moreover, a meta-analysis has shown that the use of Flash glucose monitoring was associated with a clinically significant reduction in 
HbA1c [24].

A critical note about CGMs is validating their performance, whereas FGM is factory-calibrated, indicating that no validation against SMBG is required. The most common metric used to assess CGMs performance is the mean absolute relative difference (MARD), which is the mean of the absolute errors between all CGMs values and the reference values. The lower the MARD is, the better the performance is [9]. However, the methodology for calculating MARD has not yet been standardized, so this would be misleading [25].

\subsection{Continuous Blood Glucose Monitoring Systems in Saudi Arabia}

In KSA, insulin pump therapy and CGMs are now available and increasing as a modality for better management of DM and as an educational tool. The new user-friendly generations of CGMs encouraged people with DM to use this technology. The initiation of reimbursement of these tools by the national health bodies and insurance organizations paved the way in front of the healthcare professional to explore the recent technologies for better glycemic control and its subsequent improvements in the quality of life of their patients. All these are reflected in reducing the cost of illness and the burden of DM. CGMs are available in Saudi Arabia with all its four categories: rtCGM like the Dexcom; FGM like the FreeStyle Libre; blinded (professional) CGM like the Guardian; and the unblinded CGM. Although CGMs have become the gold standard in managing patients who are in intensive insulin therapy, some physicians are reluctant to utilize them, most probably due to the lack of experience and knowledge with this technology.

\subsection{The Clinical Targets for CGMs Data Interpretation}

In 2019, the international panel of diabetes experts demonstrated ten metrics with their target range in the CGM data interpretation with a consensus on the TIR to complement HbA1c. These metrics include the number of days CGMs has worn (recommended 14 days); the percentage of time CGMs is active (recommend $70 \%$ of data from 14 days); mean BG; glucose management indicator (GMI); glycemic variability (\% CV); time-above-range (TAR) with two levels; time-in-range (TIR); and time-below-range (TBR) in two levels. In addition, the expert panel set the accepted target in each metric for T1DM, T2DM, and older/high-risk T1DM or T2DM, and for pregnant women with T1DM, T2DM, or gestational diabetes (GDM) [3].

The new term GMI replaced the term estimated A1C. It is based on the CGMderived mean BG in the previous 14 days [14]. In addition, different research studies showed the correlation between HbA1c and the TIR. One was conducted upon 545 patients with T1DM and the other upon 1137 patients with T1DM and T2DM [26] [27]. TIR was validated as an outcome measure for DM complications like retinopathy and microalbuminuria [28]. 


\subsection{Integrating Continuous Glucose Monitoring Systems into Clinical Practice}

CGMs provide current and future BG data and display them in numerical and graphical ways, along with glucose trends. Retrospective analysis of CGMs historical data can help in carbohydrate counting and lifestyle modifications. Moreover, some CGMs have an alert feature, which is crucial for those with frequent hypoglycemia. In addition, the data-sharing ability and trend arrows can help in better glycemic control. Each type of CGMs has its advantages and disadvantages. The accuracy, the need for calibration, the easy applicability, and the cost are among the critical factors when choosing one of them.

\section{CGMs Metrics}

\subsection{Time-in-Range}

In general, the term TIR refers to the total time spent in a target BG range (70 $180 \mathrm{mg} / \mathrm{dL})$ or the more strict range $(70-140 \mathrm{mg} / \mathrm{dL})$ in some conditions. Of course, it adds a valuable piece of information about the current level of glycemic control at a specific time. That also has led to new terms like times below range (TBR) and times above range (TAR), which gave a better quantification of the level of BG control. TIRs can help people with DM watching the improvement or deterioration in the amount of clinically significant hyperglycemia or hypoglycemia over time [9] [29].

\subsection{Hypoglycemia}

In people with DM, particularly T1DM, hypoglycemia is a common complication of treatment and is a significant barrier to glycemic control. In adults with T2DM on insulin or sulfonylureas, severe hypoglycemia, defined as needing assistance, is more frequent when $\mathrm{HbAlc}$ is at the lowest or highest levels. Quantification of the risk for hypoglycemia can be carried out using the low BG index (LBGI). However, LBGI, when based on CGM data, tends to underestimate the risk to some extent [29] [30] [31] [32]. Grading hypoglycemic events is essential in managing DM, specifically when the CGM levels indicate BG levels $<54$ $\mathrm{mg} / \mathrm{dL}$ for $\geq$ two hours (Table $1 \&$ Figure 1) [9].

\subsection{Glycemic Variability}

Another important CGM metric is glycemic variability (CV). A CV $<36 \%$ means stable BG levels, and CV $\geq 36 \%$ means unstable levels [30]. The relationship between CV to DM complications, cognitive function, and quality of life has been studied and established. Therefore, it has been accepted as an important and valuable marker for glycemic control [31] [32] [33] [34]. It gives a better insight into the dynamicity of the BG levels and their fluctuations. It is a waveform process that has an amplitude, frequency, and duration. It contributes to the risks of hyperglycemia and hypoglycemia. The higher the $\mathrm{CV}$, the higher is the association to mortality in the intensive care setting [35]-[41]. 


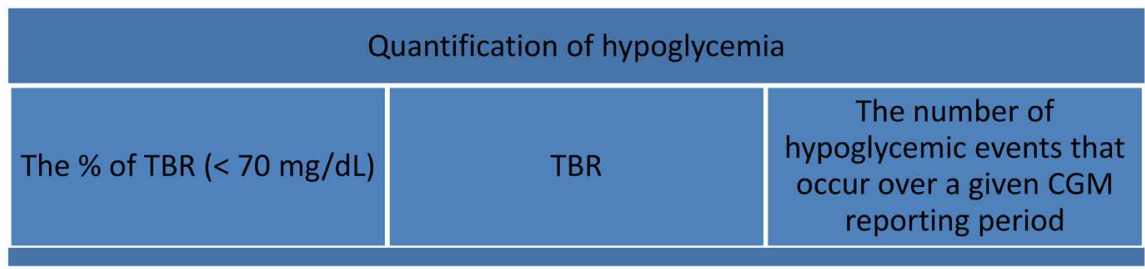

\section{Hypoglycemic event accroding to CGM data}

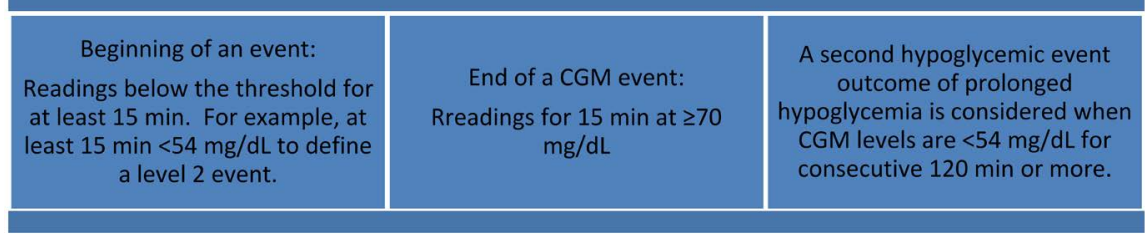

Figure 1. Quantification of hypoglycemia.

Table 1. Categorization of hypoglycemia.

\begin{tabular}{lll}
\hline Level 1 & Level 2 & Level 3 \\
\hline $\begin{array}{l}\text { BG value (70 - } 54 \mathrm{mg} / \mathrm{dL}), \\
\text { with or without symptoms. }\end{array}$ & $\begin{array}{l}\text { BG value }<54 \mathrm{mg} / \mathrm{dL}, \\
\text { with or without symptoms. }\end{array}$ & $\begin{array}{l}\text { Severe hypoglycemia, } \\
\text { denotes cognitive impairment, } \\
\text { is not defined by a specific BG } \\
\text { value. }\end{array}$ \\
$\begin{array}{l}\text { Minimization of the time spent } \\
\text { in this range will reduce the risk } \\
\begin{array}{l}\text { of developing more clinically } \\
\text { significant hypoglycemia. }\end{array}\end{array}$ & $\begin{array}{l}\text { Clinically significant } \\
\text { hypoglycemia, requires } \\
\text { immediate attention. }\end{array}$ & $\begin{array}{l}\text { Requires external assistance } \\
\text { for recovery. }\end{array}$ \\
\hline
\end{tabular}

\section{The Need for a Glucogram Similar to an Electrocardiogram}

Using standardizing reporting is beneficial in the clinical decision-making in DM management. Several reporting tools, such as the standardized Ambulatory Glucose Profile (AGP) report (Figure 2), have been developed using at least 14-consecutive-days CGM data with 70\% of readings [42] [43] [44]. Several expert panels previously adopted the AGP and recommended it as a standard tool for picturing CGM data [9] [45].

Moreover, integrating these metrics into the electronic records of people with DM is of utmost importance as they can facilitate communication with patients and help them self-manage their DM [46].

Different types of graphs help figure out the exact situation of BG control and BG distribution in one day or in a certain period in time (Figure 3(a)). These reports can be printed, certain areas of hypo or hyperglycemia can be marked and discussed with patients efficiently in relation to their daily routine (Figure 3(b)). In addition, the graphs can be daily, each day in a line graph (Figure 3(c)). In addition, another graph can show a summary and give how much time of the 14 days the device was active (Figure 2).

The four key metrics that require attention are data sufficiency (a minimum of two weeks of CGM use); the percentage of time (or minutes) on TIR, TAR \& TBR; 


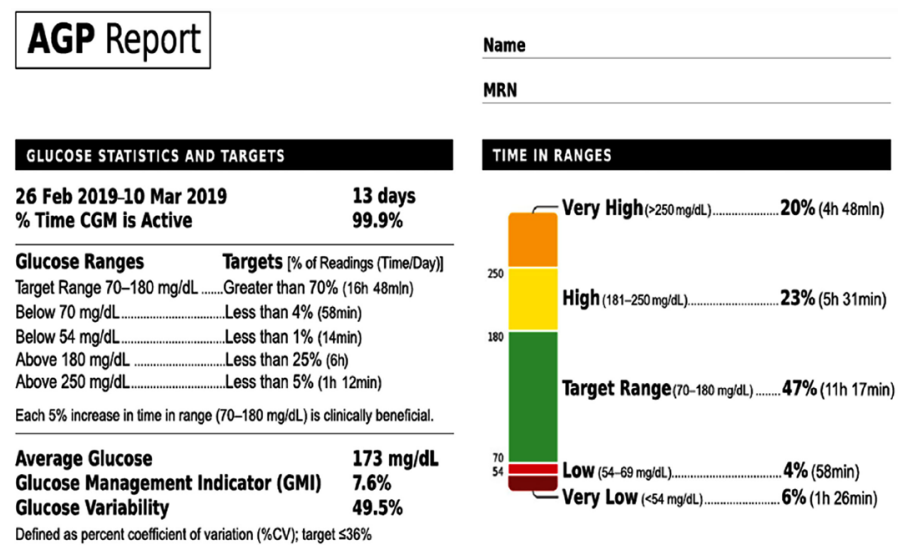

Figure 2. Ambulatory Glucose Profile (AGP) Report-Metrics and glucose pattern summary. For illustrative purposes, the outputs from the FreeStyle Libre Health Management System software (Abbott Laboratories). The Ambulatory Glucose Profile (AGP). (a2021 International Diabetes Center at Park Nicollet, Minneapolis, MN. AGPreport.org).
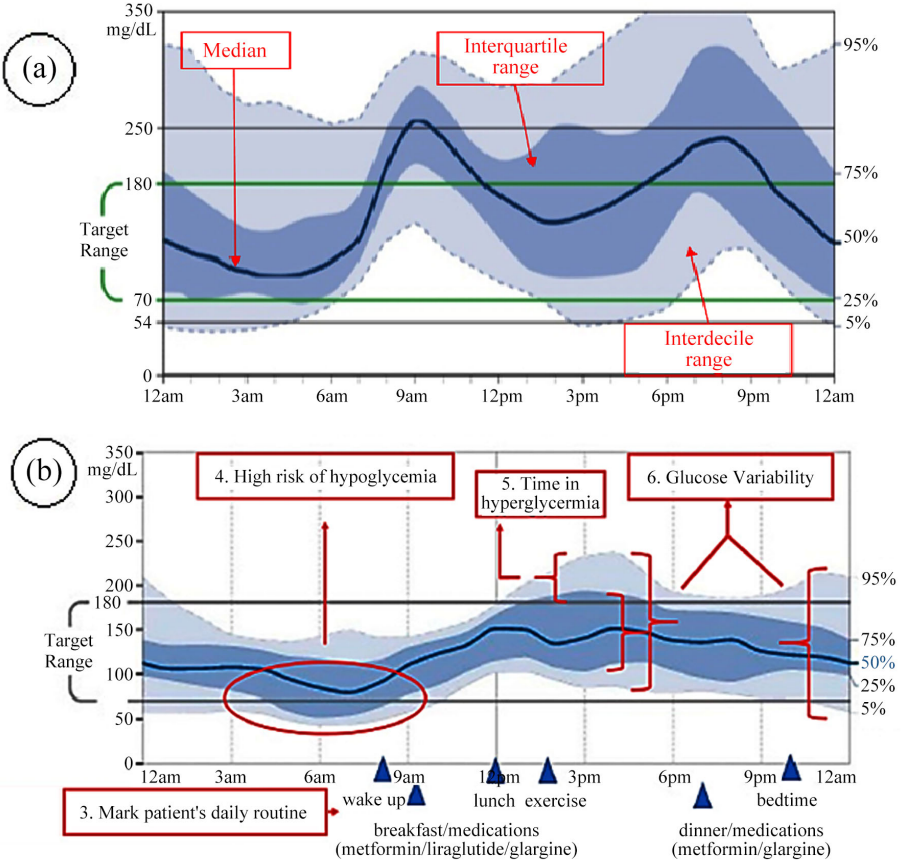

\section{DAILY GLUCOSE PROFILES}

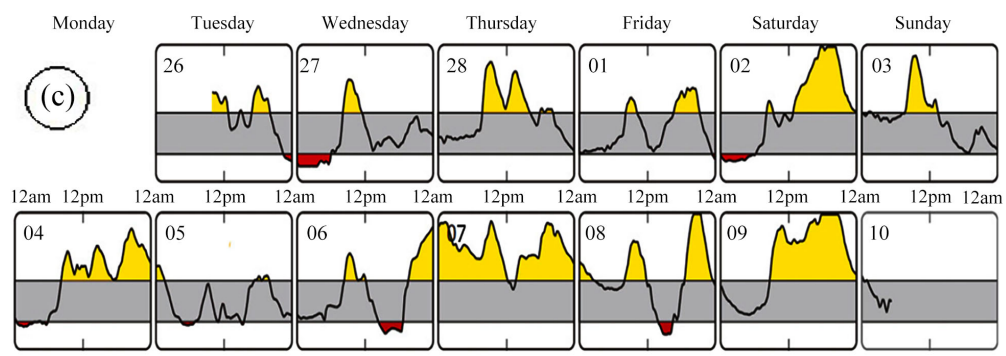

Each daily profile represents a midnight-to-midnight period.

Figure 3. Ambulatory Glucose Profile: (a) glucose distribution as if happening over a period of 24 hours; (b) Areas to target for management; (c) glucose distribution in different days. For illustrative purposes, the outputs from the FreeStyle Libre Health Management System software (Abbott Laboratories). The Ambulatory Glucose Profile (AGP). (a2021 International Diabetes Center at Park Nicollet, Minneapolis, MN. AGPreport.org). 
$\mathrm{CV}$, which indicates the level of glycemic variability over the period reported; glucose management indicator (GMI) which replaced the term estimated HbAlc. The patients using CGMs need to check their BG by fingerprick whenever there is low blood sugar, rapidly changing BG level if symptoms do not match BG reading if sensor glucose does not match BG, and confirmatory check pre-prandial. However, newer algorithms of improved accuracy would require a confirmatory fingerstick in case of symptoms not matching the readings. Training for the patients is essential for using the AGP and managing their BG accordingly. One important note mentioned is that despite no calibration is needed, still, finger-stick glucose checks are needed.

\section{Conclusions and Recommendations}

Statement 1: If we are to improve our healthcare programs in line with international evidence and technology, we need to redesign programs, re-organize and redirect our resources, and focus on our needs and goals. In diabetes care, we need to adopt new helpful technologies in an integrated, planned, shared, and structured model of care in line with the significant reform objectives of our health system.

Statement 2: HbAlc and SMBG are not enough to detect BG fluctuations daily.

Statement 3: Daily use of CGM provides the ability to obtain immediate feedback on the current level, and the trend of glucose provided by CGMs allows people with DM to act in response instantaneously and appropriately according to these data.

Statement 4: In clinical practice, metrics like TIR, TBR, TAR, GMI, and CV are valuable clinical targets that complement the laboratory HbA1c. They are an integral component of day-to-day DM management.

Statement 5: We recommend using CGMs technology in people with T1DM children and adults, T2DM on multiple insulin injections, GDM who need further glycemic control, those at high risk for hypoglycemia. In addition, we recommend using them for a short period for those who require intensive BG control or during acute illness or stress. In addition, AGP could be used as an educational tool for any individuals with DM to study the impact of some aspects of lifestyle modifications on their immediate BG level.

Statement 6: The incorporation of CGMs technology like FreeStyle Libre with its applications like Libre View is now used in many institutes in Saudi Arabia. This system is comprehensive and has all standardized metrics needed. However, training and support are always needed. In addition, two complementary ways are needed; one is the clinical evidence of its benefits, and the second is its impact on the budget (is it cost-saving?).

Statement 7: Barriers and challenges include the awareness \& experience of the technology, the time-barrier, the patients' barriers, the technical barriers, and of course, the availability barrier. All the barriers and challenges should be dealt with by designing new training programs. 


\section{Conflicts of Interest}

The authors declare no conflicts of interest regarding the publication of this paper.

\section{References}

[1] International Diabetes Federation (2017) IDF Diabetes Atlas. 8th Edition, International Diabetes Federation, Brussels. http://www.diabetesatlas.org

[2] Al Dawish, M.A., Robert, A.A., Braham, R., Al Hayek, A.A., Al Saeed, A., Ahmed, R.A., et al. (2016) Diabetes Mellitus in Saudi Arabia: A Review of the Recent Literature. Current Diabetes Reports, 12, 359-368. https://doi.org/10.2174/1573399811666150724095130

[3] Battelino, T., Danne, T., Bergenstal, R.M., Amiel, S.A., Beck, R., Biester, T., et al. (2019) Clinical Targets for Continuous Glucose Monitoring Data Interpretation: Recommendations from the International Consensus on Time in Range. Diabetes Care, 42, 1593-1603. https://doi.org/10.2337/dci19-0028

[4] Kirkwood, M. (2019) New Recommendations for Time-in-Range Targets during Continuous Glucose Monitoring Presented Today at the ADA's Scientific Sessions. http://www.diabetes.org/newsroom/press-releases/2019/new-recommendations-for. $\underline{\mathrm{html}}$

[5] Kohnert, K.D., Heinke, P., Vogt, L. and Salzsieder, E. (2015) Utility of Different Glycemic Control Metrics for Optimizing Management of Diabetes. World Journal of Diabetes, 6, 17-29. https://doi.org/10.4239/wjd.v6.i1.17

[6] Hinzmann, R., Schlaeger, C. and Tran, C.T. (2012) What Do We Need beyond Hemoglobin A1c to Get the Complete Picture of Glycemia in People with Diabetes? International Journal of Medical Sciences, 9, 665-681. https://doi.org/10.7150/ijms.4520

[7] Orchard, T.J., Nathan, D.M., Zinman, B., et al. (2015) Writing Group for the DCCT/ EDIC Research Group. Association between 7 Years of Intensive Treatment of Type 1 Diabetes and Long-Term Mortality. JAMA, 313, 45-53. https://doi.org/10.1001/jama.2014.16107

[8] Holman, R.R., Paul, S.K., Bethel, M.A., Matthews, D.R. and Neil, H.A.W. (2008) 10-Year Follow-Up of Intensive Glucose Control in Type 2 Diabetes. The New England Journal of Medicine, 359, 1577-1589. https://doi.org/10.1056/NEJMoa0806470

[9] Danne, T., Nimri, R., Battelino, T., Bergenstal, R.M., Close, K.L., DeVries, J.H., Garg, S., Heinemann, L., Hirsch, I., Amiel, S.A., Beck, R., Bosi, E., Buckingham, B., Cobelli, C., Dassau, E., Doyle, F. J., Heller, S., Hovorka, R., Jia, W., Phillip, M., et al. (2017) International Consensus on Use of Continuous Glucose Monitoring. Diabetes Care, 40, 1631-1640. https://doi.org/10.2337/dc17-1600

[10] Bry, L., Chen, P.C. and Sacks, D.B. (2001) Effects of Hemoglobin Variants and Chemically Modified Derivatives on Assays for Glycohemoglobin. Clinical Chemistry, 47, 153-163. https://doi.org/10.1093/clinchem/47.2.153

[11] Ford, E.S., Cowie, C.C., Li, C., Handelsman, Y. and Bloomgarden, Z.T. (2011) Iron-Deficiency Anemia, Noniron-Deficiency Anemia and HbAlc among Adults in the US. Journal of Diabetes, 3, 67-73. https://doi.org/10.1111/j.1753-0407.2010.00100.x

[12] Nielsen, L.R., Ekbom, P., Damm, P., et al. (2004) HbA1c Levels Are Significantly Lower in Early and Late Pregnancy. Diabetes Care, 27, 1200-1201.

https://doi.org/10.2337/diacare.27.5.1200 
[13] Krhač, M. and Lovrenčić, M.V. (2019) Update on Biomarkers of Glycemic Control. World Journal of Diabetes, 10, 1-15.

https://www.ncbi.nlm.nih.gov/pmc/articles/PMC6347654/ https://doi.org/10.4239/wjd.v10.i1.1

[14] Lenters-Westra, E., Schindhelm, R.K., Bilo, H.J. and Slingerland, R.J. (2013) Haemoglobin A1c: Historical Overview and Current Concepts. Diabetes Research and Clinical Practice, 99, 75-84. https://doi.org/10.1016/j.diabres.2012.10.007

[15] Kato, N., Cui, J. and Kato, M. (2013) Structured Self-Monitoring of Blood Glucose Reduces Glycated Hemoglobin in Insulin-Treated Diabetes. Journal of Diabetes Investigation, 4, 450-453. https://doi.org/10.1111/jdi.12072

[16] Polonsky, W.H., Fisher, L., Schikman, C.H., et al. (2011) Structured Self-Monitoring of Blood Glucose Significantly Reduces A1C Levels in Poorly Controlled, Noninsulin-Treated Type 2 Diabetes: Results from the Structured Testing Program study. Diabetes Care, 34, 262-267. https://doi.org/10.2337/dc10-1732

[17] Franciosi, M., Lucisano, G., Pellegrini, F., et al. (2011) ROSES Study Group. ROSES: Role of Self-Monitoring of Blood Glucose and Intensive Education in Patients with Type 2 Diabetes not Receiving Insulin. A Pilot Randomized Clinical Trial. Diabetic Medicine, 28, 789-796. https://doi.org/10.1111/j.1464-5491.2011.03268.x

[18] Kempf, K., Kruse, J. and Martin, S. (2012) ROSSO-In-Praxi Follow-Up: Long-Term Effects of Self-Monitoring of Blood Glucose on Weight, hemoglobin A1c, and Quality of Life in Patients with Type 2 Diabetes Mellitus. Diabetes Technology \& Therapeutics, 14, 59-64. https://doi.org/10.1089/dia.2011.0116

[19] Bolli, G.B. (1997) Hypoglycaemia Unawareness. Diabetes \& Metabolism, 23, 29-35.

[20] Gold, A.E., MacLeod, K.M. and Frier, B.M. (1994) Frequency of Severe Hypoglycemia in Patients with Type I Diabetes with Impaired Awareness of Hypoglycemia. Diabetes Care, 17, 697-703. https://doi.org/10.2337/diacare.17.7.697

[21] Nathan, D.M., Genuth, S., Lachin, J., et al. (1993) Diabetes Control and Complications Trial Research Group. The Effect of Intensive Treatment of Diabetes on the Development and Progression of Long-Term Complications In insulin-Dependent Diabetes Mellitus. The New England Journal of Medicine, 329, 977-986. https://doi.org/10.1056/NEJM199309303291401

[22] Miller, K.M., Beck, R.W., Bergenstal, R.M., et al. (2013) T1D Exchange Clinic Network. Evidence of a Strong Association between Frequency of Selfmonitoring of Blood Glucose and Hemoglobin A1c Levels in T1D Exchange Clinic Registry Participants. Diabetes Care, 36, 2009-2014. https://doi.org/10.2337/dc12-1770

[23] Pickup, J.C., Freeman, S.C. and Sutton, A.J. (2011) Glycaemic Control in Type 1 Diabetes during Real Time Continuous Glucose Monitoring Compared with Self Monitoring of Blood Glucose: Meta-Analysis of Randomised Controlled Trials Using Individual Patient Data. BMJ, 343. https://doi.org/10.1136/bmj.d3805

[24] Evans, M., Welsh, Z., Ells, S. and Seibold, A. (2020) The Impact of Flash Glucose Monitoring on Glycaemic Control as Measured by HbAlc: A Meta-Analysis of Clinical Trials and Real-World Observational Studies. Diabetes Therapy, 11, 83-95. https://doi.org/10.1007/s13300-019-00720-0

[25] Ajjan, R.A., Cummings, M.H., Jennings, P., Leelarathna, L., Rayman, G. and Wilmot, E.G. (2018) Accuracy of Flash Glucose Monitoring and Continuous Glucose Monitoring Technologies: Implications for Clinical Practice. Diabetes and Vascular Disease Research, 15, 175-184. https://doi.org/10.1177/1479164118756240

[26] Beck, R.W., Bergenstal, R.M., Cheng, P., et al. (2019) The Relationships between Time in Range, Hyperglycemia Metrics, and HbAlc. Journal of Diabetes Science 
and Technology, 13, 614-626. https://doi.org/10.1177/1932296818822496

[27] Vigersky, R.A. and McMahon, C. (2019) The Relationship of Hemoglobin A1C to Time-In-Range in Patients with Diabetes. Diabetes Technology \& Therapeutics, 21, 81-85. https://doi.org/10.1089/dia.2018.0310

[28] Beck, R.W., Bergenstal, R.M., Riddlesworth, T.D., Kollman, C., Li, Z., Brown, A.S., et al. (2019) Validation of Time in Range as an Outcome Measure for Diabetes Clinical Trials. Diabetes Care, 42, 400-405. https://doi.org/10.2337/dc18-1444

[29] Rodbard, D. (2009) Interpretation of Continuous Glucose Monitoring Data: Glycemic Variability and Quality of Glycemic Control. Diabetes Technology \& Therapeutics, 11, S55-S67. https://doi.org/10.1089/dia.2008.0132

[30] Monnier, L., Colette, C., Wojtusciszyn, A., et al. (2017) Toward Defining the Threshold between Low and High Glucose Variability in Diabetes. Diabetes Care, 40, 832-838. https://doi.org/10.2337/dc16-1769

[31] Devries, J.H. (2013) Glucose Variability: Where It Is Important and How to Measure It. Diabetes, 62, 1405-1408. https://doi.org/10.2337/db12-1610

[32] Hirsch, I.B. (2015) Glycemic Variability and Diabetes Complications: Does It Matter? Of Course It Does! Diabetes Care, 38, 1610-1614. https://doi.org/10.2337/dc14-2898

[33] Service, F.J. (2013) Glucose Variability. Diabetes, 62, 1398-1404. https://doi.org/10.2337/db12-1396

[34] Bergenstal, R.M. (2015) Glycemic Variability and Diabetes Complications: Does It Matter? Simply Put, There Are Better Glycemic Markers! Diabetes Care, 38, 1615-1621. https://doi.org/10.2337/dc15-0099

[35] Rodbard, D. (2012) The Challenges of Measuring Glycemic Variability. Journal of Diabetes Science and Technology, 6, 712-715. https://doi.org/10.1177/193229681200600328

[36] Kovatchev, B. and Cobelli, C. (2016) Glucose Variability: Timing, Risk Analysis, and Relationship to Hypoglycemia in Diabetes. Diabetes Care, 39, 502-510. https://doi.org/10.2337/dc15-2035

[37] Cox, D.J., Kovatchev, B.P., Julian, D.M., et al. (1994) Frequency of Severe Hypoglycemia in Insulin-Dependent Diabetes Mellitus Can Be Predicted from Self-Monitoring Blood Glucose Data. The Journal of Clinical Endocrinology and Metabolism, 79, 1659-1662. https://doi.org/10.1210/jc.79.6.1659

[38] Kovatchev, B.P., Flacke, F., Sieber, J. and Breton, M.D. (2014) Accuracy and Robustness of Dynamical Tracking of Average Glycemia (A1c) to Provide Real-Time Estimation of Hemoglobin A1c Using Routine Selfmonitored Blood Glucose Data. Diabetes Technology \& Therapeutics, 16, 303-309. https://doi.org/10.1089/dia.2013.0224

[39] Egi, M., Bellomo, R., Stachowski, E., French, C.J. and Hart, G. (2006) Variability of Blood Glucose Concentration and Short-Term Mortality in Critically Ill Patients. Anesthesiology, 105, 244-252. https://doi.org/10.1097/00000542-200608000-00006

[40] Eslami, S., Taherzadeh, Z., Schultz, M.J and Abu-Hanna, A. (2011) Glucose Variability Measures and Their Effect on Mortality: A Systematic Review. Intensive Care Medicine, 37, 583-593. https://doi.org/10.1007/s00134-010-2129-5

[41] Qu, Y., Jacober, S.J., Zhang, Q., Wolka, L.L. and Devries, J.H. (2012) Rate of Hypoglycemia in Insulin-Treated Patients with Type 2 Diabetes Can Be Predicted from Glycemic Variability Data. Diabetes Technology \& Therapeutics, 14, 1008-1012. https://doi.org/10.1089/dia.2012.0099 
[42] International Diabetes Center (2020) AGP_Ambulatory Glucose Profile: AGP Reports. http://www.agpreport.org/agp/agpreports

[43] Medtronic (2020) New CareLink iPro Pattern Snapshot.

https://professional.medtronicdiabetes.com/para/new-carelink_-ipro_-pattern-snap shot-report

[44] Dexcom (2020) Dexcom CLARITY Diabetes Management Software. https://www.dexcom.com/clarity

[45] Bergenstal, R.M., Ahmann, A.J., Bailey, T., et al. (2013) Recommendations for Standardizing Glucose Reporting and Analysis to Optimize Clinical Decision Making in Diabetes: The Ambulatory Glucose Profile (AGP). Diabetes Technology \& Therapeutics, 15, 198-211. https://doi.org/10.1089/dia.2013.0051

[46] Xing, D., Kollman, C., Beck, R.W., et al. (2011) Juvenile Diabetes Research Foundation Continuous Glucose Monitoring Study Group. Optimal Sampling Intervals to Assess Long-Term Glycemic Control Using Continuous Glucose Monitoring. Diabetes Technology \& Therapeutics, 13, 351-358.

https://doi.org/10.1089/dia.2010.0156 